\title{
Qualified Person Certificate License Text
}

National Cancer Institute

\section{Source}

National Cancer Institute. Qualified Person Certificate License Text. NCI Thesaurus. Code C94032.

A character string that describes the specific credentials of the qualified person. 